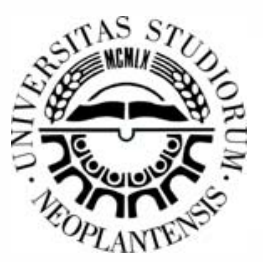

\title{
Consideration of Absorption Coefficient Changes in Numerical Simulations of Laser Forming
}

\author{
Helge Kügler $^{a, *}$, Frank Vollertsen ${ }^{a, b}$ \\ ${ }^{a}$ Bremer Institut für angewandte Strahltechnik GmbH, Klagenfurter Str.5, 28359 Bremen, Germany \\ ${ }^{b}$ University of Bremen, Germany
}

\begin{abstract}
Material processing with laser beams is well-known in nowadays production. Compared to $\mathrm{CO}_{2}$ lasers, modern solid state lasers are, amongst others, popular because of higher energy efficiency and higher absorption when metals like steel and aluminum are irradiated. However, the absorption of metals is not only dependent on the chemical composition of the work piece metal and the laser beam wavelength. Previous investigations determined the oxidation of the surface as an influence on laser beam absorption changes due to multiple irradiation. In this study, a method is presented for considering the absorption coefficient changes caused by surface oxidation in numerical simulations. Reproductions of single trajectories were assigned with appropriate absorption coefficients calculated from a function generated by reference tryouts. With the described approach, benefits are gained for numerical simulations of laser beam forming (like bending) and other processes with an iterative heat input.
\end{abstract}

Key words: laser bending; absorptivity; iterative processes; simulation accuracy

\section{INTRODUCTION}

Absorption of radiation is dependent, amongst others, on the wavelength of the radiation. For laser processing of steels this was investigated by Dausinger and Shen [1]. Other influencing factors are correlated with surface properties. Previous investigations point out that oxidation due to multiple laser processing increase the absorptivity [2].

Absorptivity is an important parameter for simulations and predictions of laser beam processes. For laser beam bending, for example, knowledge of the absorption coefficient is decisive for the prediction accuracy of bending angle calculations, which can be done by an analytical model provided by Geiger and Vollertsen [3].

Besides bending, laser radiation can also be used for supporting conventional forming processes. For example, Gisario et al. illustrated the potential of laser radiation supporting mechanical bending in order to control springback [4]. Another application for lasers in forming processes is single point increment forming with laser heating on the backside of the sheet. Duflou et al. identified benefits of additional laser heating, such as force reduction and increasing formability [5]. Additionally, less springback and a reduced stress level were achieved. These advantages were confirmed by Biermann et al. [6]. They investigated incremental titanium sheet forming with laser support on the same sheet surface as the forming tool. They showed that the maximum forming depth could be significantly increased with preheating temperatures of $400^{\circ} \mathrm{C}$. In further studies, Göttmann et al. developed a setup for laser-assisted asymmetric incremental sheet forming in which the laser beam is guided concentrical to the forming tool [7]. Advantages of laser heating could be confirmed, but temperature measurement was challenging, and they conclude that a suitable control concept is needed. Alternatively, knowledge of the absorption coefficient changes during processing would enable a laser power control to hold a constant temperature during the process. Therefore, absorptivity changes due to multiple laser irradiation have to be investigated.

* Corresponding author's.e-mail: kuegler@bias.de 


\section{EXPERIMENTAL SETUP AND METHODS}

\section{$2.1 \quad$ Laser processing}

A multiple linear laser irradiation of stainless steel sheets (X5CrNi18-10; AISI 304) with a thickness of $0.6 \mathrm{~mm}$ was carried out. The specimens were cut to a size of $50 \mathrm{~mm} \times 76 \mathrm{~mm}$.

Laser radiation was provided by a disc laser (Trumpf TruDisk 12002) and was guided by programmable focusing optics (PFO 3D with focal length of $450 \mathrm{~mm}$ ). A $50 \mathrm{~mm}$ defocused laser beam was used, resulting in a measured spot size of $3.08 \mathrm{~mm}$. The laser power was set to $250 \mathrm{~W}$ for all experiments with an additional linear ramping from $125 \mathrm{~W}$ to the target power of $250 \mathrm{~W}$ within $0.5 \mathrm{~mm}$ at the beginning and at the end of the sheet to avoid overheating and melting at the edges. The velocity of the laser beam on the steel sheet was set to $90 \mathrm{~mm} / \mathrm{s}$. Shielding gas was not used.

The amounts of laser processing cycles were varied from 1 to 100 with intermediate steps at $2,5,10,25$, and 50 . Every parameter set was carried out three times. With increasing laser processing cycles, bending of specimens occurs. In order to analyze only absorptivity changes induced by thermal surface modification and not by bending deformations, all specimens were clamped on both sides of the metal sheet (cf. Fig. 1). With this experimental procedure a master curve for the absorptivity changes due to multiple laser processing was determined. This master curve was then used as a look-up table for the absorptivity of each laser processing cycle in a numerical simulation.

\subsection{Absorption measurements}

Measurements of the absorption coefficient were carried out using an integrating sphere (cf. Fig. 2) with a BaSO4 coating. This coating is highly reflective for radiation in the near infrared spectrum. The special manufactured sphere has three ports. On the top of the sphere the low power testing laser beam is guided into the sphere and onto a specimen which is positioned at the second port at the bottom. The opening of this second port has a diameter of $5 \mathrm{~mm}$. Reflections from the specimen are mainly guided to the wall of the sphere in an angle which equals the angle of incident, which was $8^{\circ}$ in this case. The coating on the wall of the sphere causes diffuse reflection. Therefore, the radiation is assumed to be homogenized and measurements of the photo diode placed at the third port are considered to be representative.

Absorptivity of each specimen was characterized after laser processing. The irradiation was carried out with a $300 \mathrm{~mW}$ laser diode (Lumics LU1030M300). This laser diode provides radiation with a wavelength of $1030 \mathrm{~nm}$ which equals the wavelength of the processing laser. The laser diode radiation was collimated with a focal length of $2 \mathrm{~mm}$ (Thorlabs CFC-2X-B). Measurements of the reflected radiation was carried out with a silicon-based photodiode (Thorlabs FDS100, rise time: $10 \mathrm{~ns}$ ). The system was calibrated by measuring an $\mathrm{Nd}$ :YAG mirror (Thorlabs NB07-K14). According to its datasheet, the reflectance of unpolarized laser radiation of the used wavelength of $1030 \mathrm{~nm}$ with the mentioned irradiation angle of $8^{\circ}$ was $99.89369 \%$. Measured values of the photo diode for mirror irradiations were calibrated to this value.

\subsection{Simulation approach}

The software simufact.welding 6.0.0 was used for numerical simulations of a laser beam bending process. Sheet dimensions were set to the specimen size of the laser processing experiments. Meshing was carried out using Abaqus/CAE 6.14-2. A mixed meshing structure was used. In the middle of the heat source trajectory a hexahedral mesh with a structured technique was used, whereas outer regions were meshed with hexahedral dominated elements using the sweep technique. Within the fine meshed area $14 \times 100$ nodes were used. This results in a mesh size of $0.76 \mathrm{~mm}$ in $\mathrm{x}$ direction and 0.36 $\mathrm{mm}$ in $\mathrm{y}$ direction. In $\mathrm{z}$ direction, the sheet thickness was meshed with four elements. The meshed sheet is shown in Fig. 3.
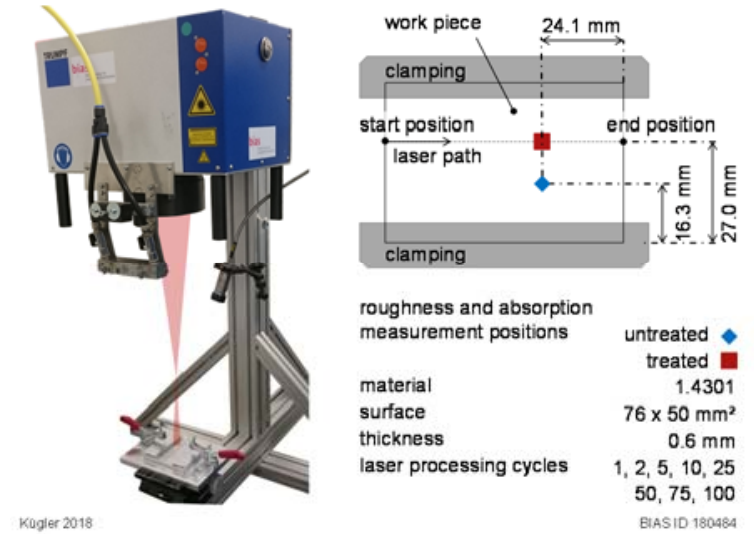

Fig. 1 Setup for multiple linear laser processing
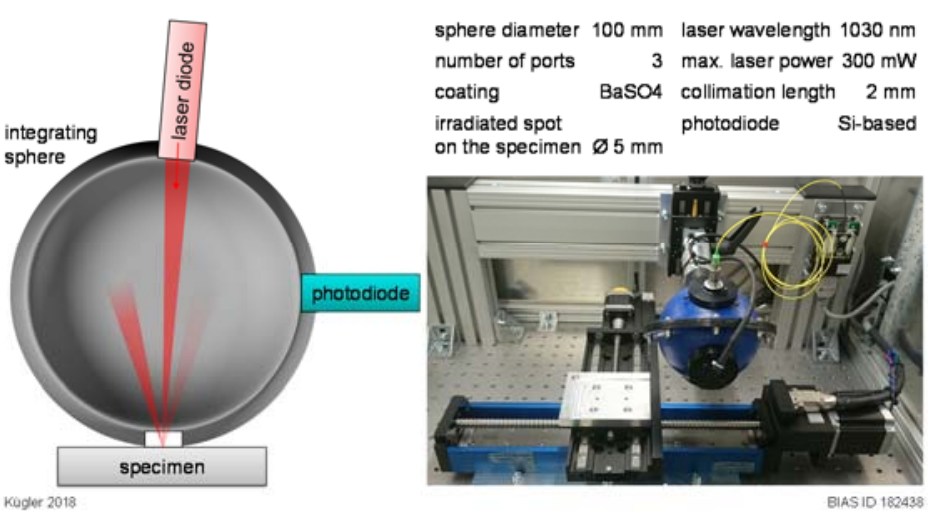

Fig. 2 Principle of the laser beam absorption measurement with an 


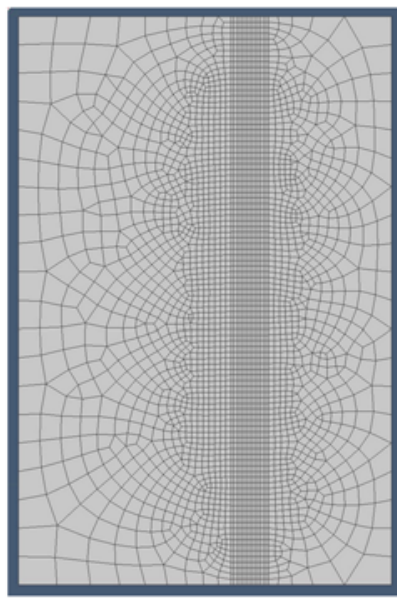

Kügler 2018

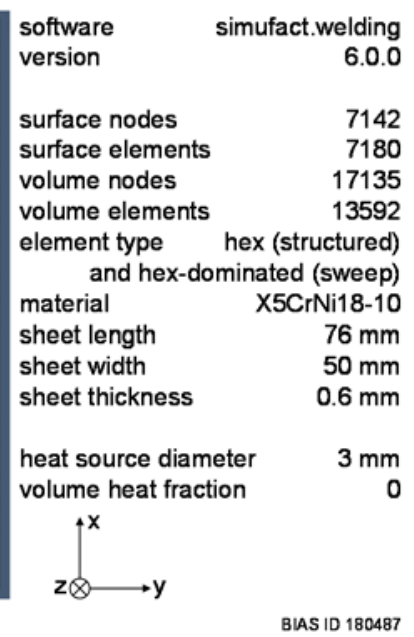

BLAS ID 180487
Fig. 3 Meshed sheet for the numerical simulation of a laser beam bending process

Heat source properties in the simulation software were set as close as possible to the measured properties of the laser beam used for laser processing. Therefore, a surfacebased heat source (volume heat fraction was set to 0 ) with a diameter of $3 \mathrm{~mm}$ was used.

In order to allow bending in the simulations, the sheet was fixed at only one side. Bending angles were calculated with the distortion values in z-direction in the middle of the sheet after the simulated laser processing duration plus an additional cooling time of $180 \mathrm{~s}$.

\subsection{Implementation of an adaptive absorption coefficient}

For the simulation of the iterative laser bending process, a single irradiation cycle trajectory with a specific heat source was designed in the software simufact.welding. Subsequently, this single cycle was duplicated to the desired amount of irradiation cycles. This reproduction was carried out by using MATLAB R2016a. Simulation projects in simfact.welding are saved in project files which can be read and edited by MATLAB or an text editing program.

For each reproduction of the trajectory build in the single cycle simulation, the initial heat source is copied and assigned. Each heat source, which is only used for one specific trajectory, has an efficiency value. This equals the absorptivity of the laser beam power on the surface of the material. Therefore, the absorption coefficient can be adjusted for the next irradiation cycle according to the master curve which was determined before and represents the relation between the amount of laser processing cycles and the absorption coefficient.

As a result of the trajectory and heat source reproduction, a new simufact.welding simulation project is generated. Processing of this new project with multiple laser irradiation cycles is identical to the single trajectory project. Therefore, the modified project can be transmitted to the standard solver used in simufact.welding. With this procedure, a customized simulation project file is generated and hence is treated as one continuous simulation. Thus, solving with the simulation software considers all effects which are considered in manual designed projects, like e.g. strain hardening or heat accumulation.

\section{RESULTS}

Irradiated specimens clearly show annealing colors on the surface (cf. Fig. 4). Due to the heat input, distortions of the steel sheets can be observed. These distortions partially result in roughness changes. Though, these roughness variations are a side effect and are not considered to cause absorptivity changes [2].

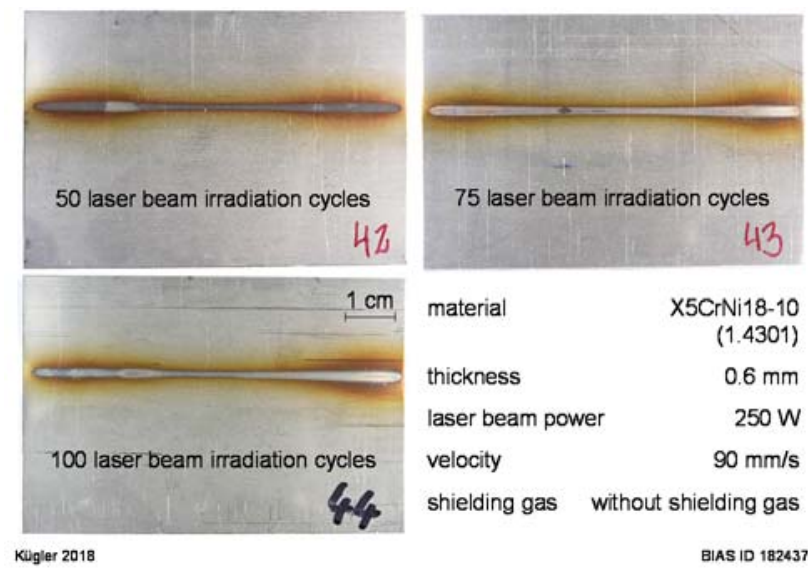

Fig. 4 Irradiated specimens with annealing colors

Measuring all specimens result in an initial absorption coefficient of $41.49 \%$. After laser processing this value is increased (cf. Fig. 5). The amount of absorptivity increase per laser irradiation cycle can be described with a linear function. Especially for up to ten laser processing cycles, standard deviations are below $4 \%$ of the measured absorptivity value. Although, standard deviations for more than ten laser processing cycles are higher, the coefficient of determination for the linear regression function is 0.904 .
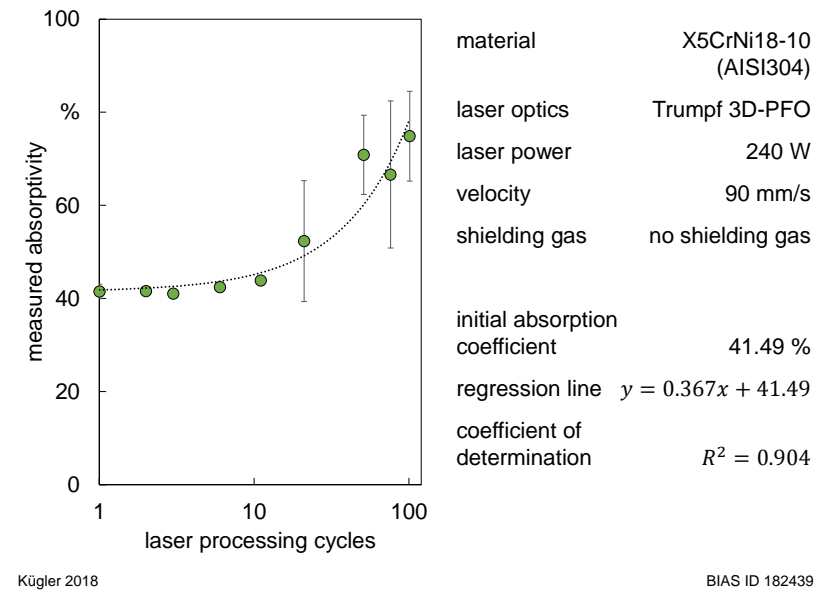

Fig. 5 Measured absorption values depending on the amount of laser processing cycles (master curve) 

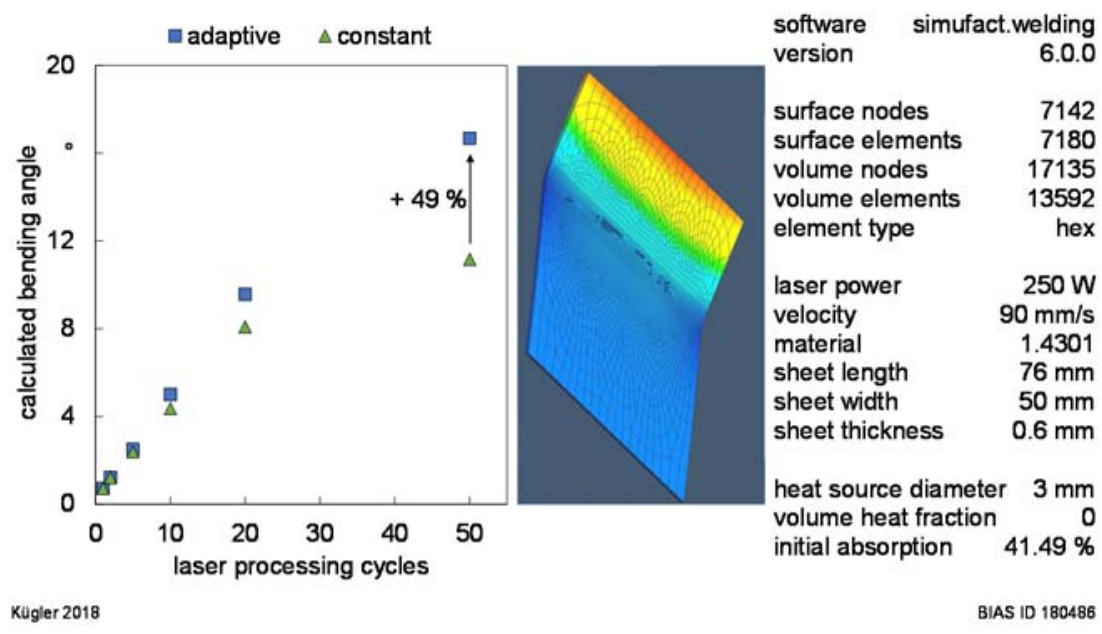

Fig. 6 Comparison of numerical simulations with constant and adaptive absorption coefficients

This linear function was used as a master curve to calculate the absorption coefficient for each cycle and implement these values into a numerical simulation with the above-mentioned method. For small amounts of laser processing cycles, resulting bending angles are nearly the same (cf. Fig. 6). For up to five laser processing cycles, simulations with an adaptive absorption coefficient result in bending angles which are $4.9 \%$ higher than bending angles of simulations with a constant absorption coefficient.

The discrepancy between a constant and an adaptive absorption coefficient is increasing with higher amounts of laser processing cycles. For 50 processing cycles the discrepancy is $49.4 \%$ (cf. Fig. 6).

\section{Discussion}

The results illustrate that the influence of previous laser processing on the absorptivity has to be taken into account even more for processes with a high amount of laser processing cycles. For up to five laser processing cycles, discrepancy of the absorption coefficient is less than $2 \%$ and therefore supposed to be neglectable. The difference in resulting bending angle simulations is less than $5 \%$.

It can be shown that a linear regression function is suitable to characterize the amount of absorptivity changes per irradiation cycle in this case. A coefficient of determination of 0.904 indicates a sufficient agreement of this master curve. Influences of the material composition and its initial structure have to be taken into account. Therefore, a reference curve like the one presented in this study (cf. Fig. 5) has to be produced.

Implementing adaptive absorption coefficients in a numerical simulation of a bending process was successful. Reproducing a single trajectory with an appropriate absorption coefficient taken from the master curve by a multi-paradigm numerical computing environment like MATLAB is a suitable method for implementing an adaptive absorption coefficient. Thus, the same solver can be used for numerical calculations and the visualization of the result as well as the simulation behavior regarding the calculation time is equal to simulations with a constant absorption coefficient.

Until now, the absorption coefficient is often used to fit simulation results to experimental results. In this case an absorptivity higher than the measured initial absorptivity of $41.49 \%$ had to be chosen in order to compensate the absorptivity changes due to laser processing. But, a simulation based on a fitted absorption coefficient can only be precise for a fixed amount of laser processing cycles and cannot be scaled to another amount of processing cycles. Furthermore, simulations with the initial state absorption coefficient used for all laser processing cycles is underestimating the increasing absorptivity.

Furthermore, this approach of implementing variations of the absorption coefficient can be used for processes with iterative laser treatments like bending or incremental forming processes. Temperature measurements can be challenging due to a limited accessibility in the working field of the forming tool. This issue was amongst others described by Göttmann et al. [7]. They carried out laserassisted asymmetric incremental sheet forming with the laser beam guided concentrically to the forming tool. The results of this study indicate that direct temperature measurement could be circumvented by generating a reference curve for the specific material and therefore enable calculations of an adaptive absorption coefficient and adjusting a desired temperature for the next forming increment. Knowledge of the absorption coefficient is mandatory in order to determine the accurate laser power in considerations of previous process steps.

\section{CONCLUSIONS}

In this study, the consideration of absorptivity changes in numerical simulations is presented. Absorptivity measurements with an integrating sphere were carried out after multiple laser beam irradiations of stainless steel 
sheets. Out of these, reference curves were produced in order to determine a function for absorptivity changes. A linear regression was identified to be suitable to describe a master curve for the absorptivity increase due to multiple laser processing in this case. Reproducing a single trajectory with an appropriate absorption coefficient taken from the master curve by a numerical computing environment like MATLAB was identified to be a suitable method for implementing an adaptive absorption coefficient in numerical simulations of iterative laser beam processes. Therefore, numerical simulation with an adaptive absorption coefficient were realized by implementing this master curve. The results indicate that differences between simulations with and without an adaptive absorption coefficient are increasing with increasing laser processing cycles. In the presented case, the difference of resulting bending angles is neglectable for up to five laser processing cycles. For numerical simulations of up to 50 laser processing cycles, significant deviations in the resulting bending angles of up to $49 \%$ were identified. Therefore, the implementation of an adaptive absorption coefficient was found to be beneficial for high cycle laser beam processes.

From these results we conclude that in simulations of processes with multiple laser irradiation of the same work piece area much more realistic results will be achieved, if the changes of the absorption coefficient by the irradiation is accounted by using an appropriate absorptivity function.

\section{ACKNOMFDGEMENTS}

Funding by the Deutsche Forschungsgemeinschaft DFG (project number 274609062) is gratefully acknowledged. The authors also thank Mr. Gustavo Eltjan Loman for performing the experiments.

\section{REFERENCES}

[1] Dausinger, F.; Shen, J. (1993). Energy Coupling Efficiency in Laser Surface Treatment. ISIJ International, 33 (9) 925933

[2] Kügler, H.; Vollertsen, F. (2018). Determining Absorptivity Variations of Multiple Laser Beam Treatments of Stainless Steel Sheets. Journal of Manufacturing and Materials Processing 2 (84) DOI: https://doi.org/10.3390/jmmp2040084

[3] Geiger, M.; Vollertsen, F. (1993). The Mechanisms of Laser Forming. CIRP Annals - Manufacturing Technology 42 (1) 301304

[4] Gisario, A.; Mehrpouya, M.; Venettacci, S.; Barletta, M. (2017) Laser-assisted bending of Titanium Grade2 sheets - Experimental analysis and numerical simulation. Optics and Lasers in Engineering 92, 110119

[5] Duflou, J. R.; Callebaut, B.; Verbert, J.; Baerdemaeker, H. de (2007) Laser Assisted Incremental Forming - Formability and Accuracy Improvement. CIRP Annals 56 (1) 273276

[6] Biermann, T.; Göttmann, A.; Zettler, J.; Bambach, M.; Weisheit, A.; Hirt, G.; Poprawe, R. (2009). Hybrid Laser-Assisted Incremental Sheet Forming Improving Formability of Ti- and Mg-Based Alloys. Proceedings of the Fifth International WLTConference Lasers in Manufacturing, LIM 2009, 273-278

[7] Göttmann, A.; Diettrich, J.; Bergweiler, G.; Bambach, M.; Hirt, G.; Loosen, P.; Poprawe, R. (2011). Laser-assisted asymmetric incremental sheet forming of titanium sheet metal parts. Production Engineering 5 (3) 263271 